\title{
Impacto de la evaluación del desempeño en la calidad de atención que brinda el personal de enfermería del Hospital de Cuilapa
}

\section{Impact of performance evaluation on the quality of care provided by the nursing staff of the Cuilapa Hospital}

\section{Como citar el artículo}

Sandoval, J,. (2019). Impacto de la evaluación del desempeño en la calidad de atención que brinda el personal de enfermería del hosptial de Cuilapa. Revista Naturaleza, Sociedad y Ambiente, 6 (1), $41-56$ DOI: https://doi.org/10.37533/ cunsurori.v6i1.40

Jessica Adalila Sandoval Mejía de Guzmán

Sub Directora de Enfermería, Hospital Regional Cuilapa "Licenciado Guillermo Fernández Llerena"

Recibido: 14 de octubre de 2019 / Aceptado: 26 de noviembre de 2019

Disponible en internet el 29 de noviembre de 2019

*Autor para correspondencia, correo electrónico: jessicasandovaldeguzman@hotmail.com

\begin{abstract}
Resumen
Enfermería es una profesión que requiere de ciertos valores específicos como vocación, empatía, generosidad, caridad, humanismo, responsabilidad, dedicación entre oros que deben ser parte de un profesional de enfermería, el hospital regional de Cuilapa constituye uno de los 44 establecidos en la red hospitalaria es regional y se ubica en las primeras 7 posiciones por su atención, complejidad y referencia. Anualmente atiende cantidades que sobre pasan los 40 mil, pacientes que asisten a la emergencia y la afluencia por diversas causas es elevada. La presente investigación pretende establecer qué papel juega la evaluación del desempeño que se le realiza al personal de enfermería frente a una atención brindada en el marco de los estándares establecidos con el objetivo de lograr la identificación de los factores condicionantes de brindar una atención de calidad, en etapas una de las etapas difícil del ser humano que es el proceso de salud enfermedad.
\end{abstract}

Palabras clave: calidad, evaluación, factores, desempeño, competencia

\begin{abstract}
Nursing is a profession that requires certain specific values such as vocation, empathy, generosity, charity, humanism, responsibility, dedication between golds that must be part of a nursing professional, the regional hospital of Cuilapa constitutes one of the 44 established in the network Hospital is regional and is located in the first 7 positions for its attention, complexity and reference. Annually attends amounts that exceed 40 thousand, patients who attend the emergency and the influx of various causes is high. This research aims to establish what role the evaluation of the performance of nursing personnel plays in relation to the care provided within the framework of the established standards with the objective of identifying the conditioning factors of providing quality care, in stages one of the difficult stages of the human being that is the disease health process.
\end{abstract}

Keywords: quality, evaluation, factors, performance, competence 


\section{Introducción}

Enfermería es una profesión altamente humanitaria, que debe tener como requisito para los profesionales que desean ejercerla valores como empatía, amor al prójimo, responsabilidad, confidencialidad, altruismo, sensibilidad, respeto entre otros que les permitan ver y tratar a las personas como un ser humano con diversas necesidades que desean ser resultas y que muchas veces no son precisamente físicas si no falta de pertenecía por sus seres amados; el Hospital Regional de Cuilapa, actualmente maneja una alta demanda de pacientes que solicitan ser atendidos por diversas patologías, por lo que es importante que se pueda evaluar cuál es el tipo de atención que se brinda por parte del personal de enfermería ante el incremento de demanda con un mínimo de recurso humano y la relación que tiene la evaluación de desempeño.

Por ello es importante lograr alcanzar los objetivos de identificar la atención que brinda el personal con énfasis en los cuidados críticos y de emergencia en el hospital regional de Cuilapa, verificar si existen factores a los que se pueda enfrentar el personal al desempeñar su actividades y funciones diarias, así como el conocer las diversas opiniones de los pacientes que solicitan ser atendidos en el servicio de emergencia. Es importante resaltar que la identificación de cada uno de ellos es un compromiso altamente relevante pues define el desempeño del personal en un entorno álgido, en el cual deben actuar de una forma rápida y oportuna ya que de esa atención depende muchas veces la vida de los pacientes.

La evaluación del desempeño constituye hoy en día una herramienta importante para el éxito de una empresa, ya que permite mediante su utilización adecuada la búsqueda de estrategias que conduzcan a los geren- tes mejorar la atención que se brinda dentro de la misma, fortaleciendo el conocimiento de los clientes internos, mediante el análisis de sus funciones, tareas o actividades, que ayudan a mejorar el desempeño del talento humano. Tomando en cuenta que no es procedimiento nuevo, pues desde hace muchos años la misma se ha utilizado con diferentes características, pero con la misma finalidad como lo afirma diversos autores como (Chiavenato, Parra, Alles, Mcgregor, Zúñiga entre otros 2005) las bases en las que se sustentaba dicha actividad esta contenían diversas matrices enfocadas en conocer e identificar el desempeño de los empleados, aunque en su mayoría buscaban la producción de sus productos para el incremento de sus ganancias.

Es necesario resaltar que la metodología utilizada en la presente investigación fue de tipo mixto, cuantitativo y cualitativo aplicando el estudio de caso, pues su propósito es la comprensión de aspectos fundamentales, con el deseo de conocer cómo funcionan las partes y su relación, por lo que se utilizó técnicas diversas como observación, recolección de datos, encuestas, evaluaciones y entrevistas; las cuales se aplicaron al personal de enfermería y pacientes para su posterior análisis y presentación de resultados. Considerando que la calidad actualmente se encuentra delimitada en los costos que los usuarios estén dispuestos a pagar sobre todo en el ámbito de la salud, es importante conocer cuál es la atención observada en un servicio de salud de carácter público.

\section{Marco Teórico}

\section{Concepto de Calidad}

Como indica Fajardo et al. (2012), la calidad de Atención, está basada en diversos componentes entre los que se puede incluir la segu- 
ridad que se le debe brindar a los pacientes, aspecto que en algunas ocasiones se ve debilitado puesto que este principio no se cumple por diversas situaciones por ello es necesario definir la seguridad como una atención de salud libre de riesgos que incluyan estándares acordes a las afecciones de los pacientes sin provocar daños agregados. Indicando que a nivel mundial se han realizado actividades y acciones encaminadas a promover los procedimientos de la salud que garanticen calidad de los mismos; la calidad es una responsabilidad de carácter social y legal que debe de ser el marco de los gobiernos a nivel mundial.

\section{Gestión de calidad hospitalaria}

"La calidad de atención médica fue motivo de preocupación social desde los tiempos remotos: practicante surgió con los primeros estados esclavistas o con la aparición de la profesión médica. Durante la edad antigua, en los estados esclavistas de Egipto y la India, existían funcionario que observaban el cumplimiento de las normas sanitarias" (Ramos 2011:40). Sin embargo, en las áreas hospitalaria este tipo de conceptos se maneja cumpliendo los estándares, en su mayoría en los que son de carácter privado, ya que cuenta con mayor recurso económico que les permite realizar una gestión de calidad basada en certificaciones internacionales, que les permite el reconocimiento y mejorar su demanda. Por ello las Enfermeras como conductoras de los servicios deben conocer los conceptos de gestionar si como ponerlos en práctica.

\section{Calidad de los servicios hospitalarios}

Con el surgimiento del concepto de nosocomio, fue preciso buscar estrategias que permitieran garantizar la continuidad de los servicios de salud con estándares de cali- dad superiores. Resaltando que se debe en gran parte al aporte de Florence Nightingale, quien aporto en país de origen las primeras acciones y estrategias que permitieran reducir las causas por las que los pacientes fallecían en la guerra de crimea. Florence desafío todo estereotipo de la mujer en esa cultura ya que con sus aportes realizo las primeras directrices para mejorar la calidad de los servicios hospitalarios existentes. En la actualidad las instituciones hospitalarias en algunos casos intentan trabajar tomando con eje la calidad con el objetivo que les permita fortalecer su atención. (Aranza, 2008).

\section{Características del servicio de calidad}

Según Aniorte (2013), algunas de las características que se deben seguir y cumplir para un correcto servicio de calidad, son las siguientes:

a) Debe cumplir sus objetivos.

b) Debe servir para lo que se diseño.

c) Debe ser adecuado para el uso.

d) Debe solucionar las necesidades.

e) Debe proporcionar resultados.

\section{Satisfacción del usuario hospitalario}

Según Sánchez (2016), la satisfacción se presenta en todo momento, esta variable se origina de acuerdo al entorno en donde el usuario está recibiendo el servicio, la percepción determinará que la persona desarrolle una sensación satisfactoria o no con respecto al proveedor del servicio de salud. Existen varios intermediarios que permiten que se produzca la percepción y por consiguiente que se desarrolle la satisfacción. Aquellos intermediarios pueden ser lo visual como lo 
es la apariencia de la estructura del hospital o consultorio, las actitudes que pueden demostrar los médicos, personal administrativo; incluso, los de limpieza y de seguridad.

Como índica De los Ríos et al., (2004), la satisfacción del usuario está íntimamente ligado a la calidad de atención por lo cual se debe de tener bien claro cada una de estas definiciones, resaltando dentro de sus conceptos 10 factores entre los que se puede enumerar:

a) La accesibilidad de los servicios

b) La disponibilidad de los recursos

c) La continuidad de los cuidados

d) La eficacia de los cuidados

e) El costo de los servicios

f) La humanización de los servicios

g) La información al paciente

h) El acceso a la información

i) La competencia profesional del trabajador de la salud

j) El entorno terapéutico

\section{Evaluación de desempeño}

Las prácticas de la evaluación del desempeño no son nuevas. Desde que una persona empleó a otra, el trabajo de ésta fue evaluado en función de la relación costo-beneficio. Tomando en cuenta que la evaluación de desempeño no es procedimiento nuevo, ya que desde hace muchos años la misma se ha utilizado con diferentes características, pero con la misma finalidad como lo afirma diversos autores, las bases en las que se sustentaba dicha actividad contenían diversas matrices enfocadas en conocer e identificar el desempeño de los empleados, aunque en su mayoría buscaban la producción de sus productos para el incremento de sus ganancias (Chiavenato, 2007:243a).

\section{Metodología}

Basados en la necesidad de dar respuesta a los problemas de la investigación, y a los objetivos planteados, se realizó un estudio de caso, se consultaron diversas fuentes bibliográficas, en relación al tema se revisaron artículos, revistas, libros, tesis, publicaciones entre otros para luego realizar un análisis de las diversas empresas o instituciones que utilizan la evaluación del desempeño como parte importante en la atención de calidad.

Se realizaron diversas acciones y tareas para poder llegar a la obtención de los resultados, como reuniones, entrevistas, observaciones y evaluaciones con el objetivo de obtener respuestas claras que orientaran a lograr los objetivos iniciales de la investigación. Dichas actividades se realizaron durante el presente estudio en diversas ocasiones en un periodo establecido, y tener una visión más completa del escenario en donde se desempeña el personal de enfermería, del hospital regional Cuilapa en los servicios de emergencia, como base para el estudio se evaluó 55 enfermeras (o) profesionales del departamento de enfermería del servicio de emergencia, quienes representan el $100 \%$ de la población además se tomó una muestra aleatoria a conveniencia de 60 paciente, en turnos rotativos mismos que fueron tomados como base a la afluencia diaria de dicho servicio.

\section{Técnicas}

Observación: la observación fue realizada durante todo el proceso del estudio 
el mismo permitió corroborar toda la información contenida en la investigación.

e) Hojas

f) Folders

Encuesta: esta técnica se aplicó, a 55 profesionales del servicio de Emergencia y 60 pacientes que solicitaron atención durante un periodo establecido con el objetivo de obtener datos correctos para la realización del análisis correspondiente.

Entrevista: se realizó en diversos horarios durante el tiempo que duro la investigación, con el objetivo de evaluar las 24 horas en las cuales puede presentarse el paciente a solicitar atención.

\section{Instrumentos}
a) Cuestionarios
b) Block de notas
c) Lapiceros
d) Cuaderno

\section{Resultados y discusión}

La presente Investigación muestra los resultados obtenidos a través de la encuesta realizada a una muestra de 60 pacientes que visitaron la emergencia del hospital durante los meses de marzo y abril, y 55 paramédicos del servicio de emergencia, a quienes se les realizo una entrevista y una encuesta en los turnos de mañana, tarde y noche con el objetivo de lograr una visión más amplia de la relación que tiene la evaluación del desempeño con la calidad de atención; se logró establecer mediante la tabulación de datos que existe un porcentaje de $89 \%$ de satisfacción del usuario con respecto a la atención que allí se brinda, en los parámetros de buena y Regular.

\section{ATENCIÓN BRINDADA}

BUENA REGULAR DEBE MEJORAR

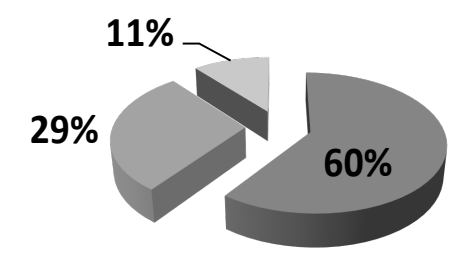

Gráfica No. 1 


\section{Opciones}

Buena

Regular

Mala

\section{Frecuencia}

36

17

7

\section{Porcentaje}

$60 \%$

$29 \%$

$11 \%$

Tabla No. 1

Se realizó un análisis de los datos obtenidos en la pregunta que se les realizó a los usuarios, en donde se pretendía investigar como definían ellos la atención que se les brindo indicando un $60 \%$ que fue buena un $29 \%$ que fue regular y un $11 \%$ indico que debería mejorar.
Como refiere Tigani (2006), un cliente satisfecho manifiesta su satisfacción, la mala atención genera motivos para ser difundidos, lograr un cliente nuevo es mucho más difícil que conservarlos motivados. Tratarlos con amabilidad, respeto y afecto genera confianza en la empresa, y borrara cualquier imagen inapropiada de las experiencias que se hayan vivido anteriormente.

\section{TIEMPO DE ESPERA}

$\square$ BUENA $\square$ REGULAR $\square$ DEBE MEJORAR

Gráfica No. 2

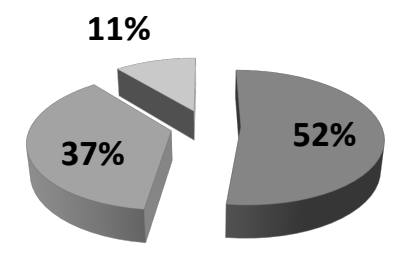

\begin{tabular}{ccc}
\hline Opciones & Frecuencia & Porcentaje \\
Buena & 31 & $52 \%$ \\
Regular & 22 & $37 \%$ \\
Mala & 7 & $11 \%$ \\
\hline
\end{tabular}

Tabla No. 2

Con relación al tiempo de espera $52 \%$ se considera entre los límites esperados, 37\% regular y un $11 \%$ indico que debían mejorarse los mismos. Evidenciado un buen porcentaje de satisfacción.

Según Palacin (2005) los tiempos de espera y su relación con la satisfacción del usuario
(2009), manifiesta que muchas veces las satisfacciones de los usuarios están íntimamente ligados al tiempo que debió esperar al momento de ser atendido, mismos que define su regreso o que busquen alternativas privadas. 


\section{ATENCION DE ENFERMERIA}

$\square$ BUENO $\square$ REGULAR $\square$ DEBE MEJORAR

Gráfica No. 3

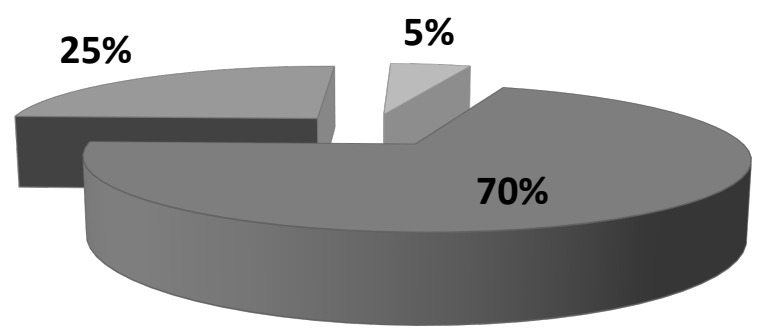

\begin{tabular}{ccc}
\hline Opciones & Frecuencia & Porcentaje \\
Buena & 42 & $70 \%$ \\
Regular & 15 & $25 \%$ \\
Mala & 3 & $05 \%$ \\
\hline
\end{tabular}

Tabla No. 3

En base a la atención de enfermería el forma que utilizan las enfermeras de cuidar $70 \%$ índico sentirse satisfecho ya que el al ser humano sano o enfermo, indicando personal mostro una actitud adecuada al que cuidar es un arte basada en principios momento de su atención, el $25 \%$ lo catalo- y postulados sustentados en el marco mego como regular pues manifestaron situa- todológico y científico, establecido como ciones y el $5 \%$ indico que debe de mejorar. un proceso que debe garantizar la calidad.

Como define Mena et al., (2016), la atención de enfermería, está relacionada a la

\section{ACTITUD DE ENFERMERIA}

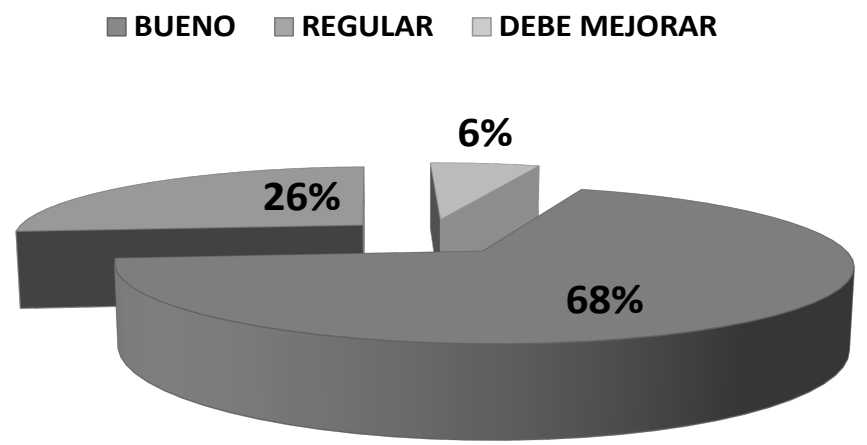

Gráfica No. 4 


\begin{tabular}{ccc}
\hline Opciones & Frecuencia & Porcentaje \\
Buena & 41 & $68 \%$ \\
Regular & 16 & $26 \%$ \\
Mala & 3 & $06 \%$ \\
\hline
\end{tabular}

Tabla No. 4

En cuanto a la actitud del personal de enfermería al momento de la atención indicaron $68 \%$ que era una buena actitud pues fueron atentos y amables con ellos, el $26 \%$ como regular pues observaron actitudes poco apropiadas y el $6 \%$ que debe de mejorar.

Como indica Leonard (2004), un buen servicio ya no basta si se desea el éxito total en una institución. Describe que la alta competitividad, que las empresas enfrentan hoy en día debido a la oferta y la demanda definen los niveles que se deben de proponer para lograr posesionarse en el mercado, resaltando que una buena actitud de los empleados garantiza el crecimiento y mantenimiento de las empresas, así como asegura sus ganancias

\section{IDENTIFICACIÓN DE ENFERMERIA}

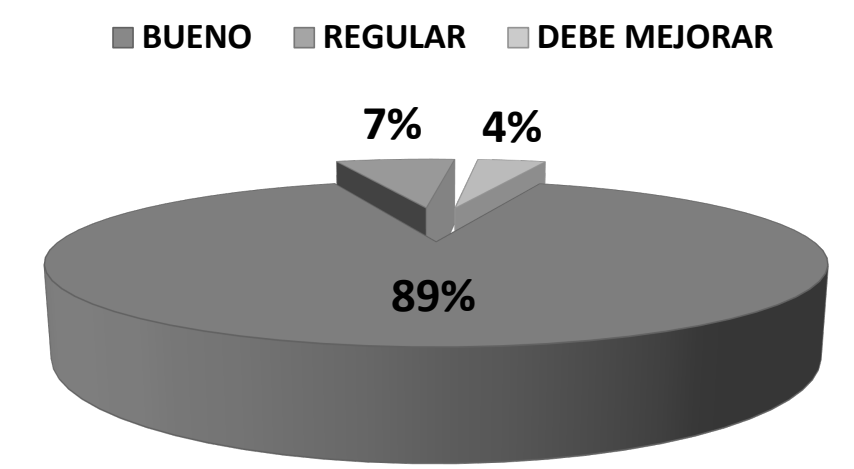

Gráfica No. 5

\begin{tabular}{ccc}
\hline Opciones & Frecuencia & Porcentaje \\
Buena & 53 & $89 \%$ \\
Regular & 4 & $07 \%$ \\
Mala & 3 & $04 \%$ \\
\hline
\end{tabular}

Tabla No. 5

En cuanto al tema de la identificación del personal el $89 \%$ coincidió que la persona indico su nombre al momento de ser atendido, un $7 \%$ indico ser regular y un $4 \%$ que debe de mejorar. Resaltando un alto índice como satisfactorio ya que es una norma en dicho personal presentarse con el paciente. La comunicación, escrita, hablada y escuchada, define las competencias y las característi- cas que tiene un líder, el contar con personal altamente capacitado y empoderado permite a la empresa mayor asertividad y tiene un impacto positivo, favoreciendo sus ingresos, así como su rentabilidad. La comunicación es una habilidad innata en algunas personas, por ello es importante tener un vínculo con los usuarios que permita confianza entre ambos (González, 2011). 


\section{CONTROL DE SIGNOS VITALES}

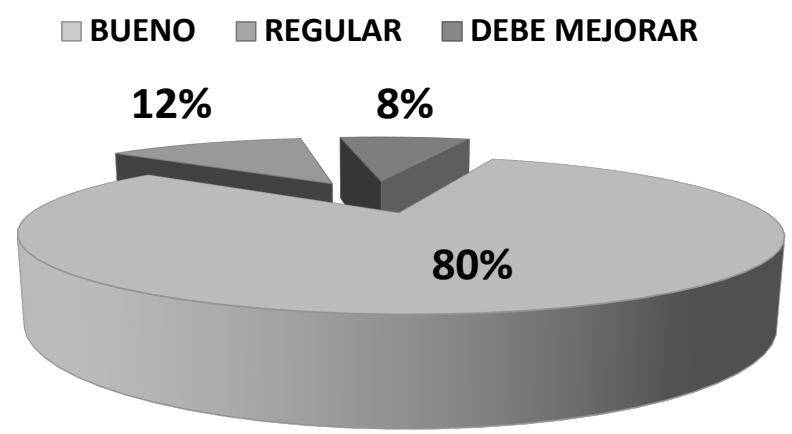

Gráfica No. 5

\begin{tabular}{ccc}
\hline Opciones & Frecuencia & Porcentaje \\
Buena & 48 & $80 \%$ \\
Regular & 7 & $12 \%$ \\
Mala & 5 & $08 \%$ \\
\hline
\end{tabular}

Tabla No. 5

Respecto al control de los signos vitales un porcentaje bastante elevado índico que fue satisfactoria la atención al momento del control de los mismos únicamente el $8 \%$ índico que se debe mejorar, pues les controlaron pulso, respiración, temperatura.

Como indica Costa et al., (2005), el conocimiento del control de signos vitales permite al personal de enfermería y de la salud, tener parámetros específicos, así como identificar señales de alarma que de ser tomados en los momentos precisos contribuyen a salvar o reestablecer la salud de los pacientes.

ASISTENCIA Y PUNTUALIDAD

$\neg$ EXELENTE $\square$ MUY BUENO $\square$ BUENO $\square$ REGULAR $\square$ DEFICIENTE

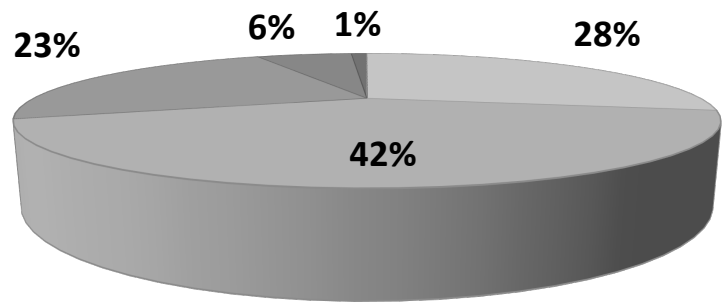

Gráfica No. 6 


\begin{tabular}{ccc}
\hline Opciones & Frecuencia & Porcentaje \\
Excelente & 15 & $25 \%$ \\
Muy bueno & 23 & $42 \% \%$ \\
Bueno & 13 & $23 \%$ \\
Regular & 3 & $06 \%$ \\
Deficiente & 1 & $01 \%$ \\
\hline
\end{tabular}

Tabla No. 6

Con relación a la asistencia y puntualidad factor que va ligado a la responsabilidad se pudo determinar que el $28 \%$ lo realizan de una forma excelente, el $42 \%$ muy bueno el $23 \%$ bueno el $6 \%$ regular y un $1 \%$ deficiente.

Es importante resaltar que enfermería es una carrera altamente disciplinaria y desde la formación de los estudiantes se resaltan estos aspectos por lo cual los mismo, des- de sus inicios traen este valor bien arraigado pues de lo contrario son sancionados según los procedimientos administrativos. La puntualidad es valor individual de los seres humanos, define y caracteriza la responsabilidad de los profesionales. Está basada en la motivación que tenga la persona y en la formación que no ha recibido. Tanto la puntualidad como la asistencia son actitudes que define a las personas (Ortiz, 2004).

\section{COMPETENCIAS}

$\square$ EXELENTE $\square$ MUY BUENO $\square$ BUENO $\square$ REGULAR $\square$ DEFICIENTE

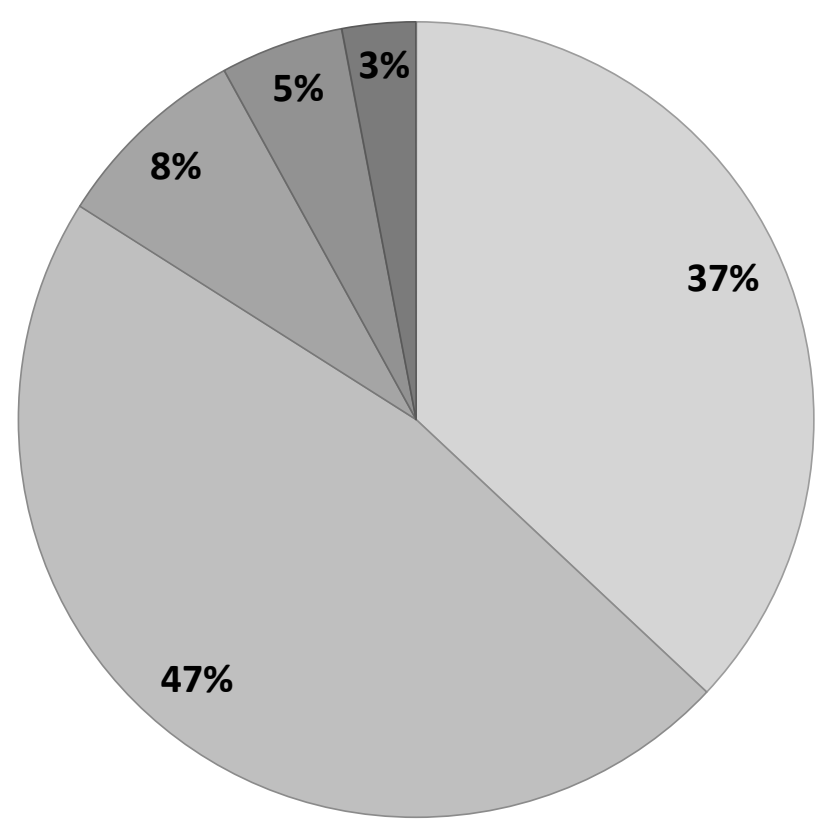

Gráfica No. 7 


\begin{tabular}{ccc}
\hline Opciones & Frecuencia & Porcentaje \\
Excelente & 20 & $37 \%$ \\
Muy bueno & 26 & $47 \% \%$ \\
Bueno & 4 & $08 \%$ \\
Regular & 3 & $05 \%$ \\
Deficiente & 2 & $03 \%$ \\
\hline
\end{tabular}

Tabla No. 7

El Profesional de enfermería tiene que fundamentar sus acciones mediante actividades netamente técnicas que favorezcan la recuperación de los pacientes a quien tiene a cargo, por ello es importante su constante capacitación y deseo de superación profesional.

Según la evaluación un $37 \%$ de los evaluados se encuentran en el rango de excelente un $47 \%$ muy bueno un $8 \%$ buen un $5 \%$ regular y un $3 \%$ es deficiente.

Como define Mertens (2000), la competencia laboral, es la destreza o capacidad que muestra un profesional ante una situación en específica; Describe que hay diferentes personas con distintas competencias y en algún momento se ven afectadas por los inconvenientes laborales negativos. Que deben ser tomados en cuenta por los clientes.

\section{Conclusiones}

Se logró observar que la atención que brinda el personal de enfermería en el hospital regional de Cuilapa en el servicio de emergencia, pese a las diversas falencias detectas el personal atiende dentro del estándar de calidad pues fueron evaluados entre 70 y $80 \%$ al momento de preguntar a los usuarios como evaluaba la atención recibida.

Durante la investigación se logró observar que existen dentro de la institución diversos factores que dificultan el que se pueda brindar una atención eficiente y efectiva entre ellos se puede listar:

a) Infraestructura reducida

b) Equipo deficiente

c) Material restringido según demanda

d) Sobre población

e) Tipos de pacientes con patologías complicadas entre otros.

Al analizar minuciosamente la encuesta que se realizó al usuario del 71 a $80 \%$ de los sujetos de estudio manifestare que recomendaría la atención de este hospital y que si fuera necesario regresaría para ser atendidos, el porcentaje restante manifestó diversos factores como tiempos de espera prolongados y afluencia de pacientes.

Según los datos de la evaluación del desempeño que fue realizada por la jefe de la sub dirección de enfermería, resaltaron que dicho proceso cualitativo tiene influencia positiva en la atención de calidad brindada a los usuarios, pues de ello depende muchas veces la diversas sanciones que realiza esa sub dirección los cuales se ven reflejadas con acciones como la recesión de contratos o inicio de procedimientos administrativos al momento de que el personal tenga calificaciones deficientes. 


\section{Referencia}

Achaerandio, L. (2010). Competencias Fundamentales para la vida: (Guatemala): Impresos IGER Talleres.

Aguilar, A. (2006). Edición y Desarrollo personal. (México): Limusa.

Albrecht, K. y B. Lawrence (2005). Excelencia en el Servicio. (Madrid): 3R 1998

Alfaro, M. (2012). Administración del personal. (México): Editorial Tercer milenio.

Alles, M. (2005). Evaluación del Desempeño por competencia. (Buenos Aires):Ediciones Granica S.A.

Alles, M. (2006). Desempeño por competencias, Evaluación de 3600. (Argentina): Editorial, Granica.

Alles, M. (2006). Dirección Estratégica de Recursos Humanos y Gestión por Competencias. (Argentina): Ediciones Granica.

Alles, M. (2007). Dirección Estratégica de Recursos Humanos. (Argentina): Editorial Ediciones Granica S.A.

Alles, M. (2008). Desempeño por Competencias. (Argentina): Editorial Ediciones Granica S.A.

Alles, M. (2009). Diccionario de Competencias. (Argentina): Editorial Ediciones Granica S.A.

Alligood, R. y A. Marrriner (2007). Modelos y teorías en enfermería. (España): Universidad de Murcia.

Andrade, J.et al (2013). "Actitud del personal profesional de Enfermería: frente a la voluntad anticipada de la persona sobre su cuidado al final de la vida." Revista electrónica enfermería actual en Costa Rica.

Angulo, Y. (2008). Ética y Valores. (México): Santillana.
Anllo, G. (2004). Evaluacion del desempeño. (Argentina): Editorial Naciones Unidas CEPAL.

Aranaz, J. (2008). La Calidad en los servicios Hospitalarios. (España): Ediciones Día de Santos.

Arellano, R. y V. Espinoza (2004). Administración de Recursos Humanos para el alto desempeño. (México): Trillas.

Arias, F. (1989). Administración de Recursos Humanos. (México): Editorial Trillas.

Arias, G. (1999). Administración de recursos Humanos. (España).

Arratia, A. (2005). Investigación y documentación histórica de enfermería. (Chile): Editoriales Ciencias Médicas.

Arriaga, R. (2010). Profesional de Enfermería. (México): RAE-Ediciones Legales.

Audrey, J. et al., (2013). Fundamentos de enfermería, conceptos, procesos y prácticas. (España): Pearson Educación.

Baenz, F. et al., (2009). Significado del cuidado en la práctica profesional de enfermería. (Colombia): Novena Edición.

Barbosa, S. (2007). Alternativas en psicología. (Brasil).

Barrios, M. (2014). "Calidad en los servicios de salud un reto ineludible". Revista cubana hematología y hemoterapia. Vol. 30, No.2, pp. 179-183.

Barrios, M. (2014). "Calidad en los servicios de salud: un reto ineludible". Revista $\mathrm{Cu}$ bana Hematología, Inmunología y Hemoterapia, Vol. 30, No.2, pp. 8-9.

Berry, L. (2004). Un buen servicio ya no basta. Editorial Deusto. (España): Ediciones Deusto.

Blanco, A. (2007). Atención al cliente. (España): Pirámide. 
Bohlander, A. y S. Snell (2003). Administración de Recursos Humanos. (España): Thomson.

Bonilla, G. (2017). Gerencia Administrativa. (Guatemala): Serviprensa.

Bonilla, G. (2019). Investigación Científica. (Guatemala): Serví Prensa.

Camison, C. et al., (2013) Gestion de la calidad, conceptos, enfoques, modelos y sistemas (Madrid): Educación S.A.

Cano, M. (2004). "Florence Nightingale, la primera gran teoría de Enfermería". Revista Cubana de Enfermería. Vol 20, No.3, pp. 3-5.

Capuano, A. (2004). "Evaluación de desempeño por competencias". Revista científica AM Latina. Vol.7, No.13. pp.139-150.

Carrillo, L. (2015). "Capacitación una herramienta de Fortalecimiento". Revistas de las sedes universitarias de Costa Rica. Vol. 16, No.33, pp.1-25.

Chávez, J. (2000) Evaluación del Desempeño. $1^{a}$ Edición. (Guatemala). Cimgra: Centro de Impresiones Gráficas.

Chiavenato, I. (2000) Administración de Recursos Humanos. $5^{a}$ Edición. (Colombia): McGraw Hill.

Chiavenato, I. (2004) Gestión del Talento Humano. (Colombia) Editorial McGraw Hill.

Chiavenato, I. (2007). Administración del Recurso Humano. (Colombia): Mc Graw Hill.

Chirinos, Y. (2018) "El clima organizacional en el emprendimiento sostenible". Revista ADM. Vol.4, No.84. pp. 436- 438.

Costa. J. y S. Rodríguez (2005). Signos Vitales. (Argentina): Guest editorial.

Cuadrado, I. (2010). "Las cinco etapas del cambio". Revista capital humano.Vol.2,
No.241. pp.56-58.

Domínguez, B. (2011). Control de calidad de la Atención de Salud. (La Habana): Ecimed Editorial ciencias Médicas.

Domínguez, P. (2008). Introducción a la gestión empresarial. (España).

Empresa pública de emergencia sanitaria, (2017). Cuidados de enfermería en la atención extra hospitalaria (España).

Encina, G. (2003). Labor y agrícola, cultivando la producción del personal. (USA).

Eseverri, C. (1992). Enfermería Hoy: Filosofía y Antropología de una profesión. (España): Investigaciones ANDINA.

Espinosa, A. (2016). "El desempeño de los profesionales de Enfermería". Revista Cubana de Enfermería, Vol 32, No 4, pp. 1-3.

Estrada. W. (2007) Servicio y atención al cliente (Perú).

Fajardo, G. (2015) Teoría y Práctica de la Administración de la Atención Médica y de Hospitales. (México).

Fajardo, G. et al., (2012). Definiciones y Conceptos fundamentales para el mejoramiento de la salud. (México): Secretaria de Salud.

Ferguson, T. (2007). Como pueden ayudar a mejorar la salud. (California): Society for participatory Medicine.

Fernández, M. (2008). Objetivos de Aprendizaje: Una forma de pensar la enseñanza universitaria. (España): Congreso iberoamericano universitario.

Fernandez, N. (2000). Fundamentos de enfermería parte 1. (Cuba): Editorial ciencias médicas.

Fernández, N. (2006). Fundamentos de Enfermería parte I. (Cuba): Ciencia Médica. 
Ferré, T. (2003). El cliente y el consumidor, protagonistas del mercado. (España): Editorial Océano.

García, A. et al., (2001). Historia de enfermería. (España): McGraw-Hill: Madrid.

García, C. et al., (2001). Historia de enfermería (España): Elsevier.

Giraldo, C. (2004). Creación de un modelo de evaluación del desempeño bajo la teoría de competencias. (Colombia): Ediciones Uninorte.

Gonzales, S. (2010). Habilidades de la comunicación escrita. (Estados Unidos): Grupo Nelson.

González, S. (2011) Habilidades de comunicación Escrita: Asertividad + persuasión + alto Impacto. (USA): Grupo Nelson.

Grinspun, D. (2002). Guía práctica en enfermería. (Canadá): Canadian.

Hernández, B. (2009). El Significado del cuidado en la Práctica Profesional de Enfermería. (Aquichan).

Isidro, R. (2013). "Importancia de la Calidad del servicio al cliente". Reviste el buzón de Pacioli. Vol.3, No.82, pp.13-15.

Kawata, P. (2009). Desarrollo de liderazgo. (USA).

Kuerten, P. et al., (2009). El cuidado y la enfermería. (Brasil): UFSC.

Ledesma, M. (2004). Fundamentos de Enfermería. (México): Limusa S.A.

Leonard, B. (2004). Un buen servicio ya no basta. (España): DEUSTO S.A.

López, L. et al., (2001). Guía para la administración segura de medicamentos (España): Editorial vista alegre.

Lozano, L. (2007). Administración de negocios. (Colombia).
Luissier, N. (2011). Liderazgo, aplicación y desarrollo de habilidades. (México): Cosegraf.

Martínez, E. (2009). Principios y Métodos. (Chile).

Martínez, E. y F. Martínez (2009). Capacitación por competencias principios y métodos. (Chile): OECD.

Martínez, M. (2011). Historia del cuidado del enfermo. (España).

Mena, D. et al., (2016). Cuidados Básicos de Enfermería. (España): I'UNE.

Ministerio de Educación. (2007). Manual de evaluación del desempeño. (Chile): Editorial Quebecor Word S.A.

Ministerio de salud. (2001). Manual de indicadores hospitalarios. (Perú).

Narey, B. y R. Domínguez (2011). Control de calidad de la alimentación de salud. (Cuba): Ciencias Médicas.

Organización panamericana para la salud (1991). La Garantía de Calidad. Acreditación de Hospitales para América Latina y el Caribe. Madrid (España).

Palacio, R. (1995). Técnicas del Servicio al Cliente. (España): Díaz de. Santos.

Peña, C. (2005). Motivación laboral como herramienta de gestión en las organizaciones empresariales. (España).

Perez, A. (2017). "Cuatro etapas del cambio organizacional". Revista cubana información en ciencias de la salud. Vol.3, No.28. pp.11-21.

Pérez, V. (2012). Calidad total en la atención al cliente. (España): Ideas propias.

Pons, S. (2016). Historia de la enfermería. (España): La RAE.

Ramos, B. (2011). Control de calidad de la atención de salud. (Cuba): Editorial Cien- 
cias Medicas.

Renteria, M. (2013). "Filosofía de la Ciencias de Cuidado". Revista Iberoamericana de las ciencias de la Salud. Vol 2, No.3, pp. 15-16.

Reyes, A. (2005). Administración de Personal. (México): Limusa.

Reyes, T. (2015). Fundamentos de enfermería. (Puerto Rico): División de Educación Continua.

Rodriguez, J. (2008). Administración moderna del personal. (México): Thomson.

Rodríguez, M. (2008). Relación médico paciente. (Cuba): Editorial Ciencias Medicas.

Saloner, S. (2008). Administración Estratégica. (México): Editorial Limusa.

Salud Pública. (2009). Tiempos de espera y la relación con la satisfacción del usuario. (Perú).

Santander, P. (2012). "Desempeño laboral de los enfermeros egresados de la universidad". Revista ciencia y cuidado. Vol.9, No.2. pp.64-70.

Sewell, C. y P. Brown (1996). Clientes para Siempre. (México): Mc Graw Hill.

Sirebrenik, J. (2003). "Que es Servqual. Medwave". Revista Biomédica. Vol.3, No.10, pp.5-6.

Sonora, I. (2013). "Importancia de la calidad del Servicio al Cliente". El buzon de Pacioli. Vol. 3, No.4, pp. 36-37.

Thomson, I.(2005) "La segmentación del Mercado" agosto 2005, pp. 1-4.

Trias, F.(2007). El libro negro del emprendedor. (Argentina): Editorial Empresa activa.

Tschohl, J. (2008). El arma secreta de la empresa que alcanza la excelencia. (Mineapolis): Quinta.
Uliar, B. (2012). Liderazgo empresarial. (México): Tercer milenio.

Vargas, F. (2003). La evaluación basada en competencia OIT. (Suiza): Editorial Cinterfor.

Vélez, C. (2011). "El cuidado en Enfermería". Revista hacia la Pas. Vol.16, No.2, pp.175-189.

Werther, W. y Keith, D. (2000). Administración de Personal y Recursos Humanos. Humano Resources and Personnel Management. (México): Editorial McGraw Hill. 


\section{Sobre autora}

\section{Jessica Adalila Sandoval Mejía de Guzmán}

Licenciada en Enfermería Colegiado número 1196 del Colegio de Profesionales en Enfermería de Guatemala. Egresada como Licenciada en Enfermería de la Universidad Mariano Gálvez y de Maestra en Artes en Gerencia Administrativa de Recursos Humanos de la Universidad de San Carlos de Guatemala, Centro Universitario del Sur Oriente CUNSURORI. Laboro Actualmente en como Sub Directora del Departamento de Enfermería del Hospital Regional de Cuilapa "Licenciado Guillermo Fernández Llerena" y como Coordinadora Académica y Docente de los cursos de Administración Hospitalaria, Enfermería pediátrica, materno infantil, Farmacología, Educación y Promoción para la Salud desde hace 8 años para la universidad Mariano Gálvez de Guatemala, asesora de tesis y monografía y supervisora de prácticas.

a) Participación en Panel Forrum como conferencista Estudiantes de Maestría UMG Jutiapa

b) "Evaluaciones de Desempeño" Marzo 2018

c) "Trabajo en Equipo" Septiembre 2018

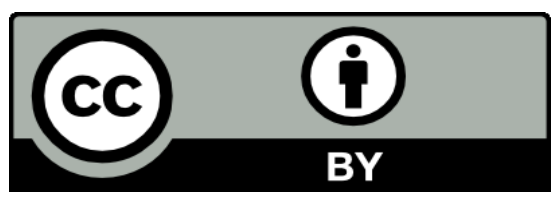

Este texto está protegido por una licencia CreativeCommons 4.0.

Esta licencia permite que otros distribuyan, mezclen, adapten y desarrollen su trabajo, incluso comercialmente, siempre y cuando le den crédito por la creación original. 